\title{
Human Language Technology can modernize writing and grammar instruction
}

\author{
Gerard Kempen \\ University of Leiden \\ P.O. Box 9555, 2300 RB Leiden, The Netherlands \\ kempen@rulfsw.leidenuniv.nl
}

The recently published Survey of the State of the Art in Human Language Technology does not spend a single word on computer-aided language learning (CALL). Indeed, present-day CALL systems hardly employ Natural Language Processing (NLP) techniques. Reasons for this state of affairs are not hard to find. First of all, current language teaching methods tend to emphasize oral language skills, especially in second-language instruction. But automatic recognition of speech, in particular speech by non-natives, has only taken its first steps outside the laboratory; and many language teachers still judge synthesized speech of insufficient quality to serve as a model for language learners. Secondly, modern language pedagogy stresses communicative success rather than formal correctness. This, too, works against the profitable deployment of NLP tools in CALL because automatically generating non-trivial, communicatively interesting and instructive dialogues does not yet seem within reach of NLP technology, let alone the evaluation of student responses from the point of view of successful interpersonal communication. More congenial with these priorities were multimedia innovations. If anything has brought about a metamorphosis in second-language teaching practices, it was the introduction of affordable video, audio and other graphical and acoustic tools that, under the control of flexible software, can create an illusion of 'total immersion'- the supposedly ideal language learning situation.

However, language proficiency includes more than conversational skills alone. Equally important are writing skills: orthography, formulating well-formed sentences, composing clear and well-organized texts. It is in the area of written language instruction that NLP technology can find a wealth of extremely valuable applications. Teaching first- and second-language writing skills is very laborintensive because teachers need to mark large numbers of test papers. NLP software holds the potential of alleviating this burden considerably, and even of outperforming teachers in the speed and quality of feedback to learners, and in the capability of generating well-targeted and attractive exercises.

A particularly important reason why human language technology should begin to take written language instruction seriously, derives from the following argument. Many instructional scientists subscribe to the view that language skills are best acquired in a situation similar to that of children learning their mother tongue. This explains not only the bias in favor of oral and conversational language skills in current language pedagogy but also the reluctance to work with explicit grammar rules. The negative attitude toward grammar is strengthened by the generally disappointing outcomes of grammarbased language teaching methods.

However, these negative results may have a completely different origin. Although the reasoning that language acquisition can do without explicit rules may hold for oral language proficiency, there is no evidence that it generalizes to the acquisition of written language skills. If writing skills do require the application of explicit orthographic, morphological, syntactic, etc., rules by the learner, then antigrammar attitudes must be detrimental. Learners will be deprived of knowledge that in fact is essential to solving writing problems. More serious is the ensuing lack of interest in the improvement of grammar instruction methods. Taught by age-old methods, many learners only have an inkling of the meaning of important grammatical concepts. Grammar rules referencing these terms are hard to apply successfully in written composition.

The response by most linguists and instructional scientists to this state of affairs has been misguided. Instead of initiating research into improved grammar teaching methods, they have tended to play down the importance of grammar rules and linguistic awareness in learning how to write. 
Modern NLP techniques for the analysis and generation of written language, in combination with graphical tools for visualizing and manipulating the structure of words, sentences and texts, afford excellent possibilities for creating integrated curricula for grammar and writing instruction. Language engineers, take up the challenge, seize the opportunity! 